\title{
Diseño de un modelo para la estimación de la dosis recibida por los colectivos cercanos a los pacientes sometidos a tratamiento de restos de Ca. de tiroides con $\left.\right|^{131}$
}

\section{Design of a model for the estimation of the ambient equivalent dose received by the closest groups to the patients undergoing treatment of remains of thyroid $\mathrm{Ca}$. with ${ }^{131}$ |}

\author{
Juan Miguel Granado Olmedo, Cristina González Ruiz, Juan Miguel Becerro Morgado, \\ Álvaro Soza Marañón
}

Hospital General Universitario Gregorio Marañón. C. Doctor Esquerdo, 46. 28007 Madrid.

Fecha de Recepción: 28/01/2020 - Fecha de Aceptación: 03/08/2020

El estudio trata de estimar adecuadamente la dosis equivalente ambiental recibida por los colectivos más cercanos al paciente, una vez éste dispone del alta radiológica y clínica.

En primer lugar, se modela un patrón de vida diaria del paciente con su entorno más cercano fijando una tasa de dosis de $40 \mu \mathrm{Sv} / \mathrm{h}$ como valor máximo medido a 1 metro para proporcionarle al paciente el alta radiológica.

En segundo lugar, se formula una ecuación de ajuste que nos proporcione la evolución de la tasa con la distancia. Tras medir a 45 pacientes a las distancias entre 0.4 y 2 metros se obtiene $R^{2} \geq 0.98$ para el $75.55 \%$ de los casos además de cometer un error menor que al utilizar la ecuación del documento del Foro sobre protección radiológica en un 93.33\% de los casos.

Al simular los modelos, se observa que las modas de las distribuciones no superan las restricciones de dosis, a saber, 3 y 15 mSv por tratamiento para acompañantes menores y mayores de 60 años respectivamente

Por último, se obtiene un tiempo de eliminación experimental del medicamento siendo menor que el dado en el documento del foro disminuyendo las modas entre la mitad y la décima parte.

Palabras clave: Tiempo efectivo de eliminación, tasa de dosis equivalente ambiental, restricción de dosis, recomendaciones post-tratamiento.

The aim is to make an adequate estimate of the ambient equivalent dose received by the groups closest to the patient, once the patient has the radiological and clinical discharge.

As a first step, a patient's daily life pattern with its closest environment has been modelled. A maximum dose rate of $40 \mu \mathrm{Sv} / \mathrm{h}$ at 1 meter has been set to give the patient the radiological discharge.

Secondly, a fitting equation that gives us the evolution of the dose rate with distance has been formulated. How the function fits to the experimental points after measuring 45 patients at the distances between 0.4 and 2 meters was verified obtaining $R^{2} \geq 0.98$ for $75.55 \%$ of the cases. Besides, a smaller error is made than using the equation of the Radiological Protection in the Healthcare Enviroment in $93.33 \%$ of cases.

When models are simulated, modes of the dose distributions do not exceed the dose restriction, namely, 3 mSv per treatment when partner is under 60 years old and $15 \mathrm{mSv}$ when companion is over 60 years old.

Finally, an experimental elimination time is obtained, being this less than the given in the Forum document, reducing the mode of distributions between a half and one tenth.

Key words: Effective elimination time, ambient equivalent dose rate, dose restriction, post-therapy recommendations.

\footnotetext{
*Correspondencia: granadoolmedo@hotmail.com

https://doi.org/10.37004/sefm/2020.21.2.001
} 


\section{Introducción}

El cáncer diferenciado de tiroides (CDT) es la enfermedad endocrina de diagnóstico más habitual $(98 \%),{ }^{1}$ aunque es un tumor poco frecuente al representar un porcentaje muy pequeño del total de cánceres detectados a nivel mundial $(<1.5 \%)$. Las expectativas del paciente, tras un tratamiento adecuado, son excelentes alcanzando tasas de supervivencia del $97 \%$ a los 5 años. ${ }^{2}$

La opción terapéutica más habitual es la extirpación quirúrgica de la glándula tiroides (tiroidectomía), combinada con la terapia metabólica, administrando 131| transcurridas 4-12 semanas desde la cirugía. ${ }^{3}$ La finalidad de este segundo tratamiento es eliminar cualquier resto tiroideo. Esta segunda fase del tratamiento se realiza en el entorno hospitalario, con una actividad prescrita según criterio clínico, proporcionando al paciente una cápsula de Nal por vía oral.

La utilidad terapéutica es debida a la emisión beta del ${ }^{131}{ }^{1},{ }^{2}$ con energía de $606 \mathrm{KeV}$, al decaer a ${ }^{131} \mathrm{Xe}$. Éste se encuentra en estado metaestable y se desexcita con la emisión de radiación gamma con energía $364 \mathrm{KeV}^{1,4}$ La emisión de radiación gamma además del porcentaje de eliminación del radionúclido a través de la orina son las razones para tener que tomar medidas de protección radiológica tras la administración del radionucleido en la terapia metabólica. ${ }^{5}$ Por ello, se ingresa al paciente en una habitación blindada con el WC conectado a un depósito especialmente preparado para recoger los excrementos en un área adecuada, lo que permite, además de la recogida controlada de la mayor parte del isotopo radiactivo eliminado por el paciente, la minimización de la exposición de los diferentes colectivos con riesgo de irradiación, particularmente el del personal sanitario y el del entorno más próximo al paciente.

Este último colectivo es el que presenta interés para nuestro trabajo, que pretende estudiar la dosis recibida por los acompañantes como consecuencia de la radiación emitida por el radioisótopo residual en el paciente tras el alta clínica, posterior al alta radiológica, en condiciones establecidas por consulta bibliográfica, tomando como referencia el documento del Foro sobre protección radiológica en el medio sanitario (CSN/SEPR/SEFM), en las que se clasifican en función de la edad del sujeto susceptible de ser irradiado. El alta radiológica se consigue cuando el paciente cumple las condiciones establecidas en el protocolo, cuya referencia es el documento del Foro.

Para menores de 60 años se restringe a 3 mSv por tratamiento, mientras que, si la edad del acompañante es superior a 60 años, las condiciones son menos restrictivas, estableciéndose la restricción en $15 \mathrm{mSv}$ por tratamiento.

\section{Material y métodos}

En el estudio se incluyen 414 pacientes diagnosticados de CDT y sometidos, tras la tiroidectomía, a un tratamiento metabólico con ${ }^{131}$ I, con diferentes actividades: 74 pacientes (17.87\%) recibieron 1110 Mbq (30 $\mathrm{mCi}), 16(3.87 \%)$ recibieron $2775 \mathrm{Mbq}(75$ $\mathrm{mCi}), 202(48.79 \%)$ recibieron $3700 \mathrm{Mbq}(100 \mathrm{mCi})$, $24(5.8 \%)$ recibieron $4625 \mathrm{Mbq}(125 \mathrm{mCi}), 1(0.24 \%)$ recibió $4810 \mathrm{Mbq}(130 \mathrm{mCi}), 84(20.29 \%)$ recibieron $5550 \mathrm{Mbq}(150 \mathrm{mCi}), 9(2.17 \%)$ recibieron 6475 $\mathrm{Mbq}(175 \mathrm{mCi})$ y $4(0.97 \%)$ recibieron $7400 \mathrm{Mbq}$ (200 mCi).

Tras la ingestión de la medicación, con el paciente hospitalizado en régimen de aislamiento en una habitación adaptada, se toman medidas de la tasa de dosis equivalente ambiental a las distancias establecidas (0.4 y 1 metro), en condiciones reproducibles, proporcionando el alta radiológica cuando este valor sea inferior a $40 \mu \mathrm{Sv} / \mathrm{h}$ a una distancia de 1 metro a la altura torácica.

La medida se realiza utilizando una mesa móvil de $88.5 \mathrm{~cm}$ de altura. El paciente se coloca en bipedestación mirando hacia el medidor. La pared más cercana al paciente se encuentra tras él a una distancia de 0.75 metros. La pared más cercana al instrumento de medida se encuentra a 1.25 metros. La altura de la habitación es de 2.5 metros.

Una vez cumplida la condición anterior, y con la certeza de que el paciente puede cumplir con las recomendaciones dadas por el Servicio de Dosimetría y Radioprotección, este recibe un documento de alta radiológica donde se detallan las recomendaciones y actuaciones que debe de seguir durante el periodo de tiempo indicado (días) una vez se encuentre en su entorno habitual. Esta información se proporciona también de manera verbal para dar la posibilidad de resolver dudas y, posteriormente, el médico podrá proporcionar el alta médica, en caso de considerarlo oportuno.

El documento entregado consta de dos partes, una de recomendaciones generales y otra en la que se especifica el periodo de tiempo de seguimiento de las mismas, ajustado a las condiciones sociales particulares del individuo, detallando en caso necesario, los 3 puntos de mayor importancia: convivencia con menores, interacciones con la pareja y comportamiento en el entorno laboral, particularizado en los casos más restrictivos (trabajo con niños o manipulación de alimentos).

El número de días de recomendaciones generales según el valor medido de tasa de dosis equivalente ambiental a 1 metro del paciente puede consultarse en el Anexo 3.B. del documento del Foro. 
En los cálculos elaborados, en Matlab R2018, para determinar la dosis que pudieran recibir los entornos cercanos de los pacientes tras el tratamiento, se consideran los días de restricción incluidos en el documento del foro para los casos más desfavorables (interacción con el acompañante) durante las 1000 horas siguientes a la salida del centro hospitalario.

Dentro de ello, se han particularizado dos modelos:

- Modelo 1: Pacientes en situación de empleo activo.

Se considera que ni el acompañante ni el paciente trabajan durante los días posteriores a la salida del hospital indicados en las recomendaciones generales, pero que, finalizado el periodo de restricción, se reincorporan a su actividad laboral. Resulta determinante indicar que la finalización del periodo de restricciones siempre es el viernes a las 23:59 horas. Esto es debido a que finalizar el periodo de restricción antes del fin de semana será el caso más desfavorable ya que durante el fin de semana el acompañante se encontrará un mayor número de horas a distancias cercanas del paciente. Cuando se le está proporcionando el alta radiológica al paciente, siempre se le indica que retome su vida normal al inicio del día siguiente en que termina el periodo de restricción. Según el modelo, se ha tomado las 11:00 horas del sábado, por lo que se da el margen de esa noche como tiempo de cautela para el inicio de la vida normal. El modelo considera que todos los pacientes finalizan su baja laboral al terminar las restricciones dadas en el alta radiológica por lo que la reincorporación al trabajo se produce el lunes a las 08:00 horas.

- Modelo 2: Pacientes sin empleo.

Se considera que ni acompañante ni paciente realizan actividades laborales fuera del entorno cercano. Al igual que en el modelo antes descrito, la finalización del periodo de recomendaciones termina el viernes a las 23:59 horas y reinicia la vida normal el sábado a las 11:00 horas.

La importancia de presentar un horario en la simulación del modelo de vida diaria del paciente en su interacción con el acompañante se debe a la disminución de la tasa de dosis equivalente ambiental con el tiempo.

Todas las simulaciones comienzan con el inicio de las restricciones para el paciente siendo a las 14:00 horas del día del alta radiológica (independientemente de la hora exacta de salida de los pacientes del hospital durante ese día). La tasa de dosis equivalente ambiental se mide una o dos horas antes del momento en que comienzan las restricciones para el paciente. Sin embargo, en los modelos se utiliza dicha tasa sin realizar la corrección por decaimiento ya que la variación es pequeña en comparación con el valor medido y nos encontraríamos en un caso más desfavorable.

Las medidas de la tasa de dosis equivalente ambiental se realizan con un instrumento portátil de medida de radiación mediante cámara de ionización presurizada de la marca Victoreen (modelo 451P-DESI-RYR NS 4427 fabricado por Fluke Biomedical). Su volumen sensible es de $230 \mathrm{~cm}^{3}$, y tiene un error en la medida $<10 \%$ y un factor de calibración emitido mediante laboratorio secundario en certificado P4170/ LMRI/GP/2521 (24 de febrero de 2017) aplicado en los cálculos.

Para los dos modelos, durante las recomendaciones, el paciente dormirá solo, por tanto, se considera que la radiación que recibe el acompañante durante las horas de sueño es cero. Salvo en las horas de las comidas en las que el paciente y el acompañante comen juntos, una hora durante el almuerzo y otra hora durante la cena, se mantendrán a una distancia variable entre 2 y 10 metros. En los modelos, durante las comidas, tomando una mesa rectangular estándar para comer dos personas como referencia, dependiendo de las posiciones elegidas por el paciente y el acompañante para sentarse en la mesa, las distancias que pueden existir entre ambos son de 0.9, 1.100 .65 metros con probabilidades asociadas del 50\%, 25\% y $25 \%$ respectivamente. Una vez hayan finalizado los días de recomendaciones, el paciente podrá dormir con su acompañante considerando una distancia constante de 0.4 m. Las horas de sueño son diferentes dependiendo del modelo y de si hablamos de fin de semana o día laboral. En común a ambos modelos, ambos duermen los fines de semana 11 horas seguidas. En el modelo 1 , los días entre semana uno de los dos trabaja en horario de mañana, por lo que las horas que coinciden de sueño son de 8 horas. En el modelo 2, de lunes a viernes ambos duermen juntos 10 horas.

En total se han realizado 8 tipos de simulaciones distintas, en función de las distancias mantenidas por el paciente y acompañante más cercano. Cada rango de éstas tendrá un valor diferente de distancia mínima entre paciente y acompañante, pudiendo ser 0.2 , $0.4,1,2,3,4,5$ o $6 \mathrm{~m}$. La máxima distancia entre ambos será fija con valor de $10 \mathrm{~m}$. La variación del espacio entre paciente y acompañante viene dada por la generación aleatoria de un número contenido en el rango de distancias de interés. Se calcula la tasa de dosis equivalente ambiental para un tiempo t, siendo durante $t$ y $t+1$ horas la dosis ambiental que recibirá el acompañante. Debido a la arbitrariedad presente en la interacción de estas dos personas, se decidió por escoger una distribución de números que tienen la misma probabilidad. 
Cada rango se simula por separado, obteniendo la distribución de dosis para cada uno de ellos. Sin embargo cuando se haga el estudio de la dosis equivalente ambiental recibida por el acompañante más cercano, se tomará el caso más desfavorable correspondiente al rango de valores comprendido entre 0.2 y 10 metros.

Es importante conocer la edad del acompañante del paciente. De este parámetro dependerá el número de días que el paciente deberá mantener las recomendaciones dadas en el alta radiológica teniéndolo que especificar en los modelos de simulación. Según las tablas del documento del Foro, si el acompañante es mayor de 60 años, ambos podrán hacer vida normal desde el momento del alta radiológica. Por el contrario si el acompañante es menor de 60 años, el número de días dependerá de la tasa de dosis equivalente ambiental medida a 1 metro del paciente.

Los datos disponibles en el momento del alta del paciente son valores de tasa de dosis equivalente ambiental a dos distancias fijas (0.4 y 1 metro), por lo que necesitamos elaborar una predicción del comportamiento de esta magnitud con su variable de mayor interés, la distancia.

El modelo más simple es considerar la fuente puntual y aceptar el decrecimiento de la tasa de dosis equivalente ambiental con el inverso del cuadrado de la distancia. Esta aproximación es poco precisa y solo se cumple a grandes distancias, cuando las dimensio- nes de la fuente son despreciables con respecto a la distancia de medida.

Por su parte, el documento del Foro propone una ecuación para un mejor ajuste relacionando la tasa de dosis equivalente ambiental con el inverso de la distancia elevado a 1.5 para distancias inferiores a 3 metros del paciente.

Si utilizamos la ecuación propuesta por el documento del foro, se produce una mejoría en el ajuste del comportamiento de la tasa con la distancia comparado con el modelo del inverso del cuadrado de la distancia. Sin embargo, pese a la mejoría proporcionada por la última ecuación, en este trabajo se presenta otra, basada en datos empíricos, cuyo comportamiento se adapta mejor a los puntos experimentales.

Buscaremos una ecuación de ajuste válida para cualquier punto de interés. Para establecerla, se realizan medidas para otras distancias $(0.4,1,1.2,1.6$ y 2 metros) con la intención de observar cómo se modifica la tasa de dosis equivalente ambiental con la distancia al paciente. Si representamos la tasa de dosis equivalente ambiental frente a la distancia al paciente en los puntos anteriores se observa en la fig. 1 el buen ajuste a una función potencia.

Por tanto, se propone una función potencia general tal y como se presenta en la ecuación (1) que nos permita relacionar la tasa de dosis equivalente ambiental en $\mu \mathrm{Sv} / \mathrm{h}, g(x)$, a una distancia del paciente en metros, $x$.

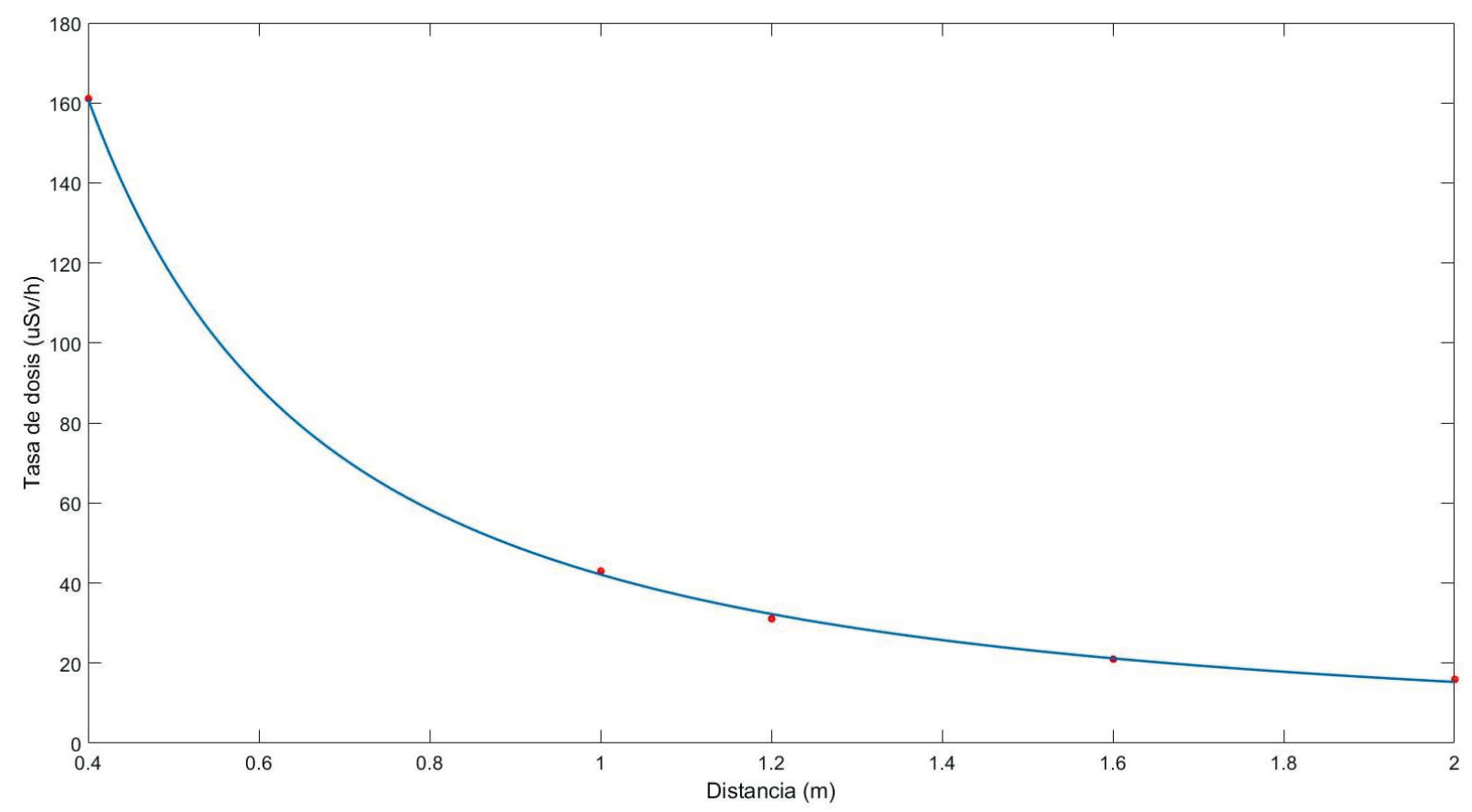

Fig. 1. Tasa de dosis equivalente ambiental frente a la distancia, para un paciente. 


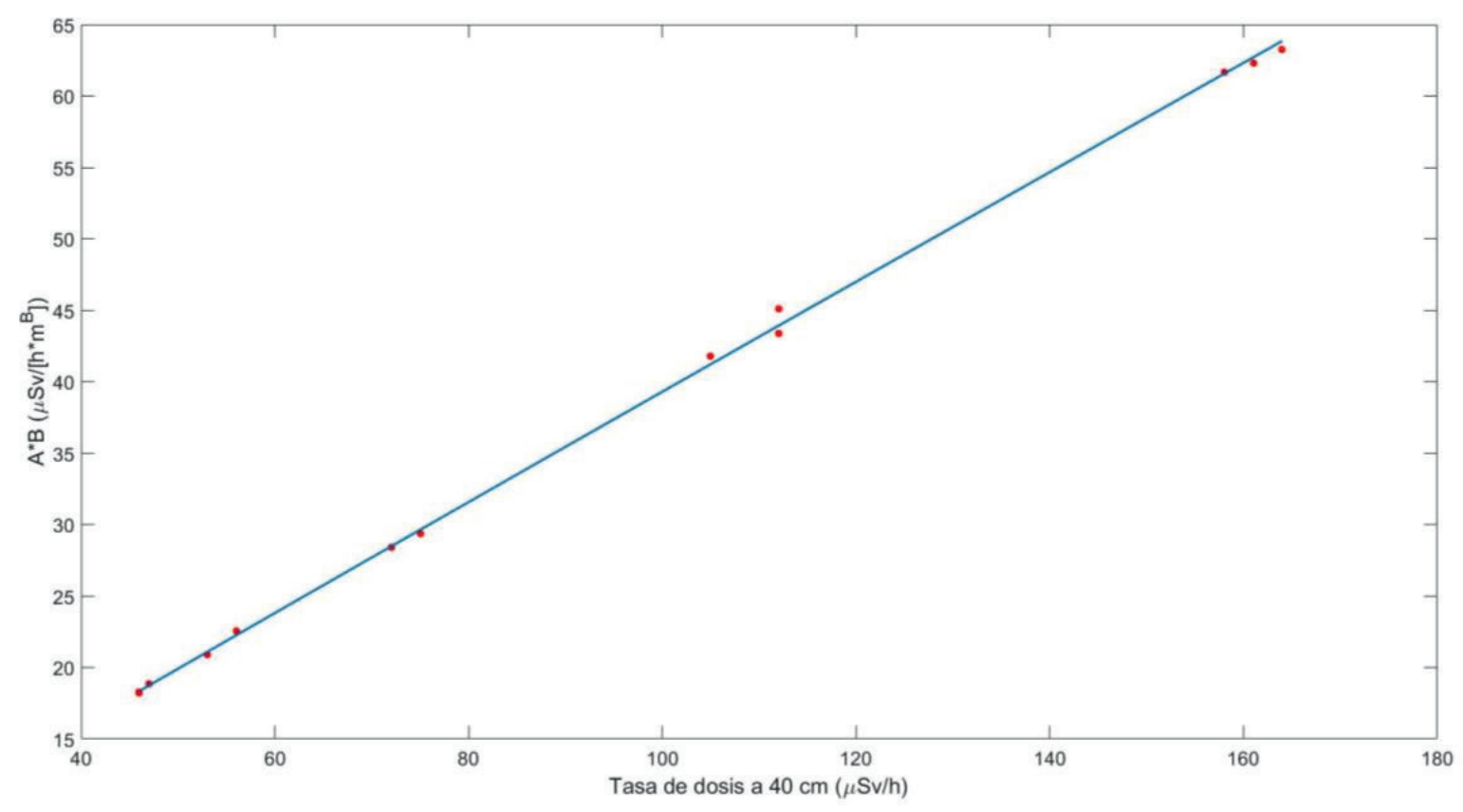

Fig. 2. Producto $A \cdot B$ frente a la tasa de dosis equivalente ambiental medida a 0.4 metros del paciente.

$$
g(x)=A \cdot x^{B}
$$

Se toman 13 pacientes para, mediante los puntos de tasa de dosis equivalente ambiental tomados a las distancias anteriores, poder escribir la ecuación (1) en función de parámetros conocidos, como son las tasas de dosis a 0.4 y 1 metro del paciente. Se representa el producto $A \cdot B$ frente a la tasa de dosis equivalente ambiental a 0.4 metros tal y como se muestra en la fig. 2. De ella se obtiene la ecuación (2).

$$
A \cdot B=0.4289 \cdot\left(\dot{D}_{0.4}\right)^{0.981}
$$

donde $\dot{D}_{0.4}$ es la tasa de dosis equivalente ambiental medida a 0.4 metros del paciente.

Si ahora representamos el parámetro $B$ frente al cociente de las tasas de dosis equivalente medidas a 0.4 y 1 metro se obtiene lo representado en la fig. 3. De ella se obtiene la ecuación (3).

donde $\dot{D}_{1}$ es la tasa de dosis equivalente ambiental medida a 1 metro del paciente.

$$
\begin{aligned}
B & =-0.0938 \cdot\left(\frac{\dot{D}_{0.4}}{\dot{D}_{1}}\right)^{2}+ \\
& +0.9089 \cdot\left(\frac{\dot{D}_{0.4}}{\dot{D}_{1}}\right)-0.6337
\end{aligned}
$$

Una vez hemos obtenido la ecuación que nos va a predecir el comportamiento de la tasa de dosis equivalente ambiental con la distancia, se realizan las simulaciones para la estimación de la dosis equivalente ambiental recibida de acuerdo a los modelos de convivencia antes definidos.

Para la simulación de las 1000 horas de vida siguientes al alta, el proceso se repite $2.5 \cdot 10^{6}$ veces, obteniendo una distribución de dosis recibida por el acompañante. Los periodos efectivos de eliminación utilizados son los propuestos por el documento base de la bibliografía, ${ }^{2} 3$ días en pacientes ablativos y 5 para pacientes con metástasis. La simulación puede ajustarse a una distribución gaussiana de forma satisfactoria, ya que los coeficientes de ajuste son cercanos a 1 para el intervalo de distancias $X-10$ metros, siendo $X$ variable 1, 2, 3, 4, 5 y 6 metros en todos los casos y modelos barajados tal como se presenta en la fig. 4 .

El coeficiente de ajuste disminuye cuando se trata de distancias más cercanas de 1 metro (0.2 y 0.4 metros), estando los coeficientes ligeramente más alejados de la unidad. Estas distribuciones de dosis se aproximan mejor a la ecuación (4), que se corresponde con una función de Gumbel, ${ }^{6}$ tal y como se muestra en la fig. $5\left(R^{2} \approx 1\right)$.

$$
\beta(x)=A \cdot e^{-\left(\frac{x-\mu}{\sigma}\right)} \cdot e^{-e^{-\left(\frac{x-\mu}{\sigma}\right)}}
$$




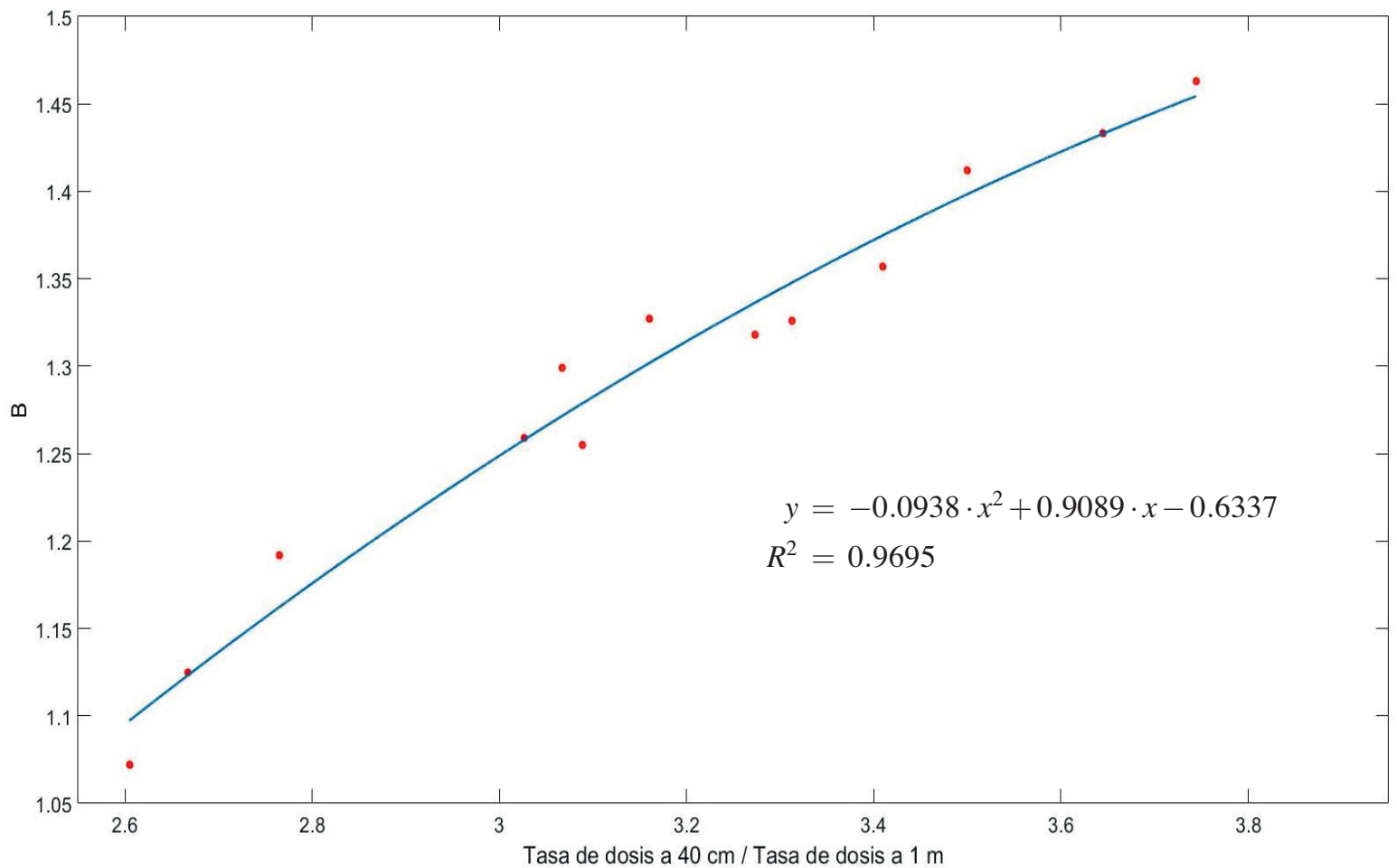

Fig. 3. Parámetro $B$ frente al cociente de tasas de dosis medidas a 0.4 y 1 metro del paciente.

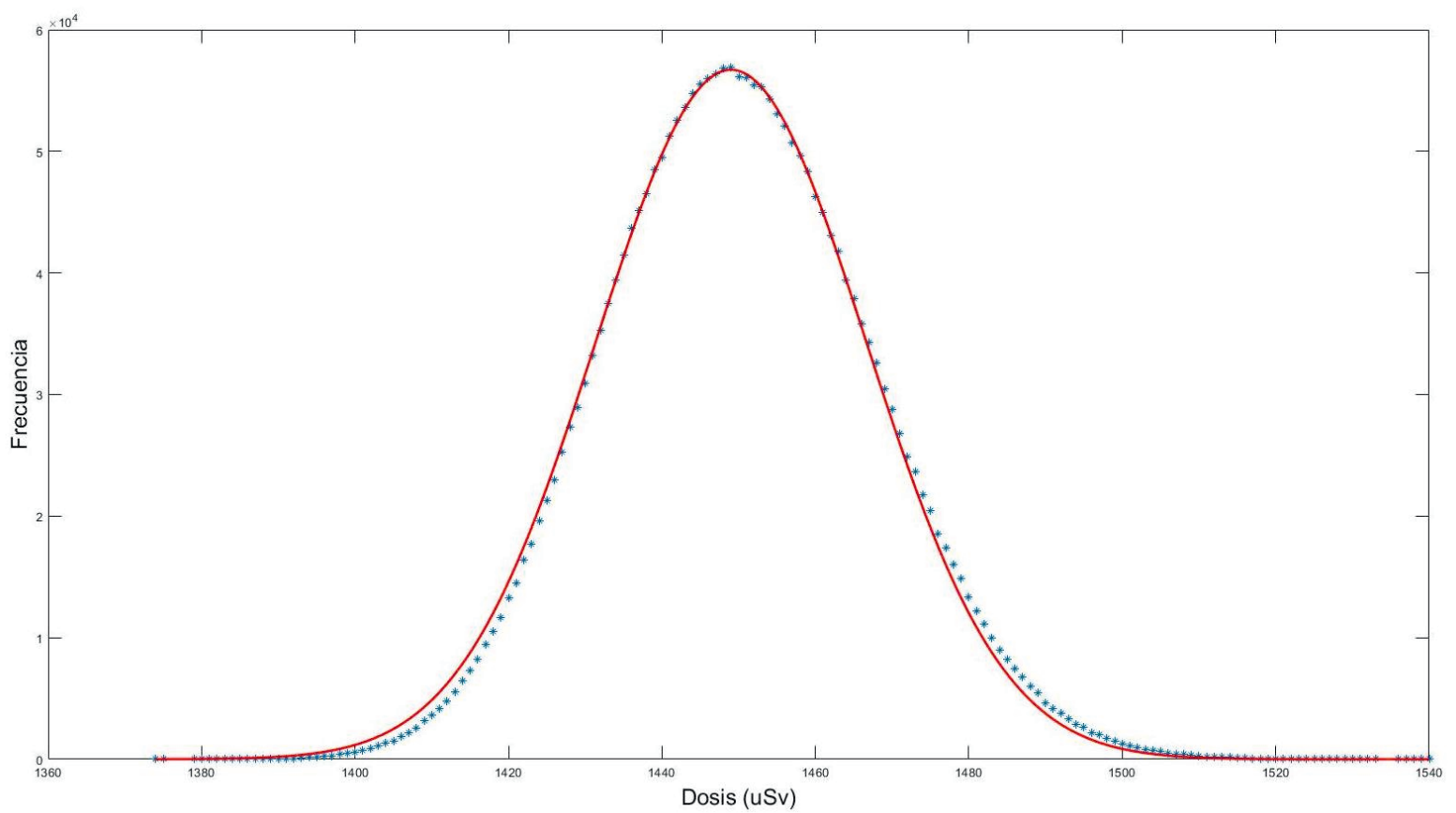

Fig. 4. Ajuste a una curva gaussiana de la distribución de dosis equivalente ambiental recibida por el acompañante para el caso de un paciente ablativo con acompañante menor de 60 años según el modelo 2. Distancias entre paciente y acompañante: 1 a 10 metros. 


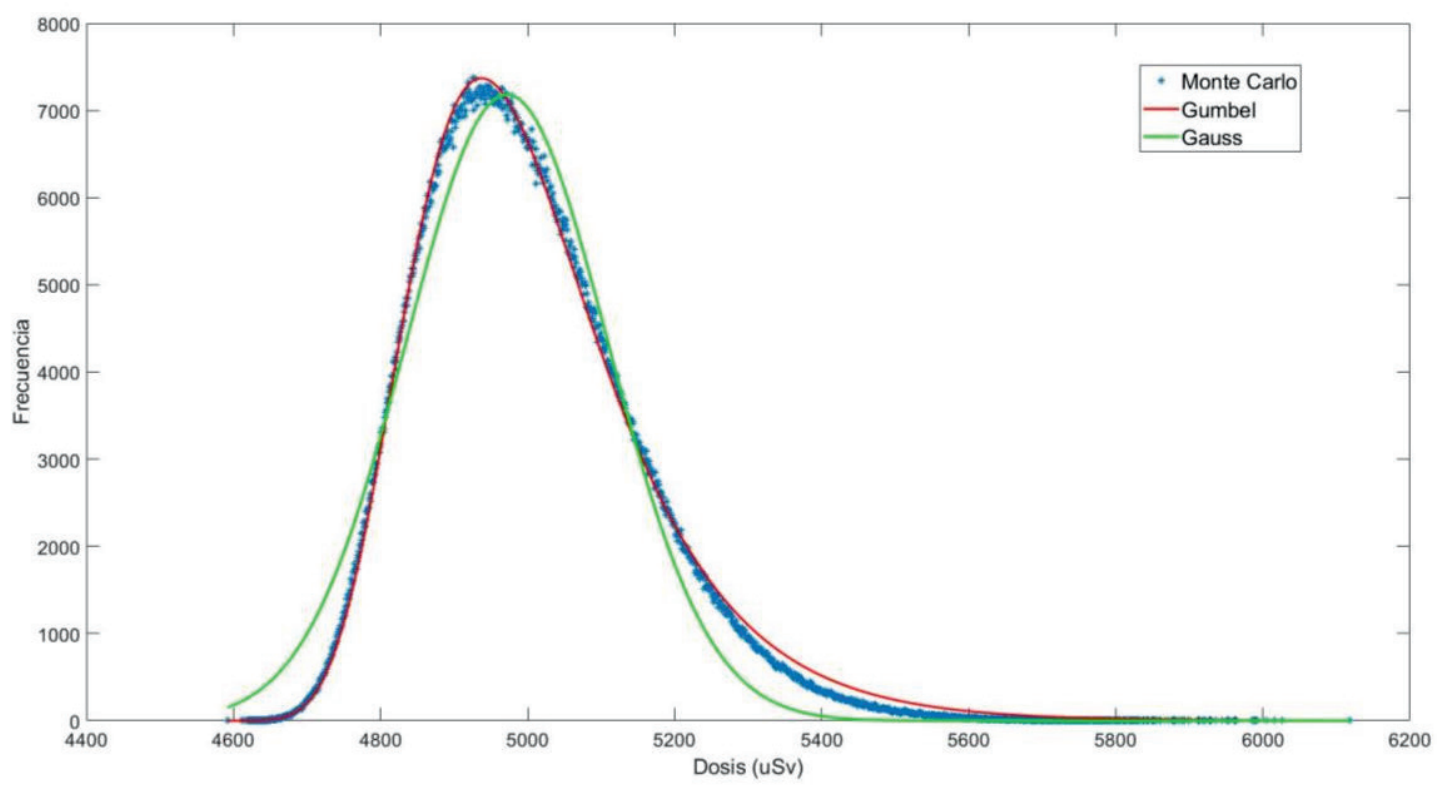

Fig. 5. Comparación entre el ajuste a la distribución de Gauss y Gumbel.

Tabla 1. Ajuste de las distribuciones de dosis a Gauss y Gumbel en función del rango de distancias entre paciente y acompañante.

\begin{tabular}{c|c|c|c|c}
$\begin{array}{c}\text { Rango de } \\
\text { distancias }(\mathrm{m})\end{array}$ & $\boldsymbol{R}^{\mathbf{2}}$ Gauss & $\begin{array}{c}\text { Desviación estándar } \\
\text { Gauss } \sigma_{\text {Gauss }}\end{array}$ & $\boldsymbol{R}^{2}$ Gumbel & $\begin{array}{c}\text { Desviación estándar } \\
\text { Gumbel } \sigma_{\text {Gumbel }}\end{array}$ \\
\hline $\mathbf{0 . 2 - 1 0}$ & 0.983 & 0.008 & 0.995 & 0.004 \\
\hline $\mathbf{0 . 4 - 1 0}$ & 0.995 & 0.003 & 0.984 & 0.005
\end{tabular}

En la tabla 1, se presentan los ajustes de las distribuciones de dosis equivalente ambiental obtenidas.

\section{Resultados y discusión}

Si tomamos como referencia la medida de tasa de dosis equivalente ambiental a $0.4 \mathrm{~m}$ del paciente y aplicamos el modelo del inverso del cuadrado de la distancia, se deduce de los casos analizados que la medida experimental es del orden del doble de la pronosticada por el modelo por lo que este se descarta.

Haciendo uso de las ecuaciones (2) y (3), la ecuación (1) se puede escribir:

$$
g(x)=\left[\frac{0.4289}{\beta} \cdot\left(\dot{D}_{0.4}\right)^{0.981}\right] \cdot x^{\{-\beta\}}
$$

donde $\beta=-0.0938 \cdot\left(\frac{\dot{D}_{0.4}}{\dot{D}_{1}}\right)^{2}+0.9089 \cdot \frac{\dot{D}_{0.4}}{\dot{D}_{1}}-0.6337$
Se introducen dos nuevos parámetros, $\delta$ y $\omega$, que harán pasar la ecuación (5) por el punto de medida a 1 metro del paciente, y reajustar el comportamiento de la función para mejorar el ajuste a los puntos experimentales. Se definen como:

$$
\begin{aligned}
& \delta=\frac{\dot{D}_{1}}{g(1)} \\
& \text { Si } \delta<1 \rightarrow \omega=\frac{1}{\delta} \\
& \text { Si } \delta>1 \rightarrow \omega=\delta
\end{aligned}
$$

Por tanto, la ecuación corregida con los parámetros anteriores que permite relacionar la tasa de dosis equivalente ambiental con la distancia al paciente será:

$$
\lambda(x)=\left[\delta \cdot \frac{0.4289}{\beta} \cdot\left(\dot{D}_{0.4}\right)^{0.981}\right] \cdot x^{\left\{-\beta \cdot \omega^{3}\right\}}
$$


Tabla 2. Media, máximo y mínimo de los errores porcentuales al comparar las medidas experimentales a $1.2,1.6$ y 2 metros del paciente con los pronosticados por las ecuaciones $f(x)$ y $\lambda(x)$.

\begin{tabular}{c|c|c|c} 
& Media & Máximo & Mínimo \\
\hline Distancia: $1.2 \mathrm{~m}$ & & & \\
\hline$f(x)$ & 20.26 & 58.21 & 1.10 \\
\hline$\lambda(x)$ & 4.74 & 20.20 & 0.18 \\
\hline Distancia: $1.6 \mathrm{~m}$ & & & \\
\hline$f(x)$ & 22.04 & 55.36 & 6.86 \\
\hline$\lambda(x)$ & 7.18 & 66.67 & 0.19 \\
\hline Distancia: $2 \mathrm{~m}$ & & & \\
\hline$f(x)$ & 21.87 & 57.81 & 2.89 \\
\hline$\lambda(x)$ & 8.31 & 87.58 & 0.11
\end{tabular}

Una vez se construyó la ecuación (8), para su comprobación, se volvieron a medir otros 45 pacientes a las distancias de 0.4, 1, 1.2, 1.6, 2 metros. El ajuste de las medidas es $R^{2} \geq 0.98$ para el $75.55 \%$ de los casos. En todos estos casos, la ecuación (8) presenta mejor ajuste que la del documento del Foro. Por tanto, este modelo es más adecuado que el propuesto por el documento de referencia.

En la tabla 2 se presentan la media, máximo y mínimo de los errores porcentuales cometidos por las ecuaciones $f(x)$ y $\lambda(x)$ al comparar los valores dados por las dos funciones y los datos obtenidos experimentalmente.

Comparando las tasas de dosis medidas con las que pronostican la ecuación (8) y la ecuación del documento del foro, $f(x)$, se observa que en 42 de los 45 casos (93.33\%) el error cometido con la ecuación (8) es menor. Además, en el $84.4 \%$, el error cometido con la ecuación (8) es menor del 10\%.

Una vez hemos obtenido la ecuación que nos va a predecir el comportamiento de la tasa de dosis equivalente ambiental con la distancia, se realizan las simulaciones para la estimación de la dosis equivalente ambiental recibida de acuerdo a los modelos de convivencia antes definidos.

En los ajustes a las distribuciones de Gauss y Gumbel, existe un valor de dosis cuya frecuencia es la mayor (moda). En la tabla 3 se muestran los valores extremos y medios de los valores de la moda dependiendo de si el acompañante o el paciente trabajan o no, modelos 1 y 2 respectivamente, y si el acompañante es mayor o menor de 60 años recopilados para todos los rangos de distancias vistos hasta el momento.

De la tabla 3, se deduce que las modas de las distribuciones de dosis recibidas por el acompañante no superan las restricciones de dosis establecidas en el documento del Foro. Sin embargo, si nos fijamos en
Tabla 3. Valores máximos, mínimos y promedios de las modas.

\begin{tabular}{c|c|c|c} 
& $\begin{array}{c}\text { Máximo } \\
(\mathrm{mSV})\end{array}$ & $\begin{array}{c}\text { Mínimo } \\
(\mathrm{mSv})\end{array}$ & $\begin{array}{c}\text { Promedio } \\
(\mathrm{mSv})\end{array}$ \\
\hline $\begin{array}{c}\text { Con trabajo mayor } \\
\text { de 60 años }\end{array}$ & 10.777 & 1.045 & 4.130 \\
\hline $\begin{array}{c}\text { Con trabajo menor } \\
\text { de 60 años }\end{array}$ & 2.470 & 0.381 & 1.671 \\
\hline $\begin{array}{c}\text { Sin trabajo mayor } \\
\text { de 60 años }\end{array}$ & 12.304 & 1.176 & 4.628 \\
\hline $\begin{array}{c}\text { Sin trabajo menor } \\
\text { de 60 años }\end{array}$ & 2.802 & 0.424 & 1.866 \\
\hline
\end{tabular}

las fig. 4 y fig. 5, se observa que las distribuciones presentan una cola de altas dosis pudiéndose dar el caso de que en ciertos casos superen el valor de restricción. De los 414 pacientes simulados, en 17 de ellos existe una probabilidad máxima de un 3.89\% de superar la restricción permitiendo un rango de interacción entre el paciente y el acompañante de 0.2 a 10 metros. Si restringimos este rango de 0.4 a 10 metros, tan solo en 3 pacientes es posible superar la restricción, con una probabilidad máxima de un $0.048 \%$.

Cuando los pacientes permanecieron ingresados más de 24 horas, se tomaron 61 medidas en pacientes ablativos y 19 en pacientes metastásicos que permitieron conocer la evolución de la tasa de dosis equivalente ambiental en los días siguientes (distanciadas aproximadamente 24 horas) pudiendo estimar un periodo efectivo de eliminación particularizado, que se presenta en la tabla 4.

Esta estimación del periodo efectivo de eliminación, también se realizó con 5 de los pacientes sometidos a terapia, tomando medidas de las tasas cuando el paciente vuelve a consulta para realizarle una gammagrafía para observar la distribución del radionucleido. Se obtuvo una tasa de dosis equivalente ambiental media de $1.9 \mu \mathrm{Sv} / \mathrm{h}$ [5.6 - 0.5]. El tiempo efectivo de eliminación medio fue de 33.1 horas [48.6 - 21.6].

Atendiendo al valor máximo obtenido para el tiempo efectivo de eliminación de la tabla 4, se pueden comparar las modas de las distribuciones de dosis recibida

Tabla 4. Máximo, mínimo, media de los tiempos efectivos de eliminación reales de los pacientes durante el tiempo de ingreso.

\begin{tabular}{c|c|c|c} 
& $\begin{array}{c}\text { Máximo } \\
\text { (horas) }\end{array}$ & $\begin{array}{c}\text { Mínimo } \\
\text { (horas) }\end{array}$ & $\begin{array}{c}\text { Media } \\
\text { (horas) }\end{array}$ \\
\hline Ablación & 31.13 & 3.17 & 17.03 \\
\hline Metástasis & 34.26 & 9.90 & 18.72
\end{tabular}


por los acompañantes del paciente utilizando este valor experimental con la derivada del cálculo realizado con el tiempo teórico. Los resultados obtenidos arrojan una diferencia notable. Para el caso de pacientes ablativos cuyos acompañantes son menores de 60 años, el valor experimental es entre 3 y 4 veces menor. Para pacientes ablativos con acompañantes mayores de 60 años la diferencia es 2 veces menor. Para pacientes metastásicos con acompañantes menores de 60 años, el valor experimental es 10 veces menor, mientras que si el acompañante es mayor de 60 años el valor experimental es entre 3 y 4 veces menor.

Como era previsible, se aprecia una notable diferencia entre los valores teóricos pronosticados y los obtenidos experimentalmente, debido fundamentalmente a que el modelo seguido en el documento bibliográfico considera tiempos de eliminación muy conservadores, acercándose el periodo efectivo de eliminación al periodo físico del radionucleido.

Esta diferencia es debida principalmente a la rápida eliminación del radiofármaco ya que solo hay captación en los restos tiroideos que quedan en el paciente después de la tiroidectomía.

\section{Conclusiones}

De acuerdo con lo presentado, la ecuación propuesta consigue la mejora de la predicción del comportamiento de la tasa de dosis equivalente ambiental a cualquier distancia inferior a 2 metros. Además, se ajusta más a las condiciones de fuente extensa a cortas distancias y fuente puntual a distancias grandes para las condiciones de vida del paciente durante el periodo de restricciones.
Los resultados de dosis obtenidos, aplicando las restricciones durante el periodo establecido, quedan alejados de los valores límite de dosis establecidos para los diferentes grupos en función de edad y características, ya que se comprueba experimentalmente que el periodo de eliminación dado por el documento del foro y usado para calcular el periodo de restricciones es mayor al obtenido tras medir a los pacientes.

\section{Referencias}

1. Ramírez-Garzón YT, Ávila O, Medina LA, Gamboa-deBuen I, Rodríguez-Laguna A, Buenfil AE et al. Measurement of radiation exposure in relatives of thyroid cancer patients treated with (131)I. Health Phys 2014;107(5):410-416.

2. Criterios de alta de pacientes y medidas para la protección radiológica del público después de tratamientos metabólicos con I-131. Foro sobre protección radiológica en el medio sanitario. Consejo de Seguridad Nuclear, Sociedad Española de Protección Radiológica y Sociedad Española de Física Médica, 2011.

3. Kochovska MZ, Jokic VS, Majstoro V, Dugonjic S. Estimated dose to family members of patients treated with radioiodine. Radiat Prot Dosimetry 2017;174(2):250-254.

4. Al-jubeh W, Shaheen A, Zalloum O. Radioiodine I-131 for diagnosing and treatment of thyroid diseases. Comunicación personal 2012.

5. Uslu-Beşli L, Demir M, Yeyin N, Çavdar I. Radiation dose of caregivers could be reduced in thyroid carcinoma patients requiring high dose of radioactive iodine: A case report. Iran J Nucl Med 2016;24(2):144-146.

6. Gorgoso-Varela JJ, Rojo-Alboreca A. Use of Gumbel and Weibull functions to model extreme values of diameter distributions in forest stands. Ann For Sci 2014;71:744-750. 


\section{FRAINLAB}

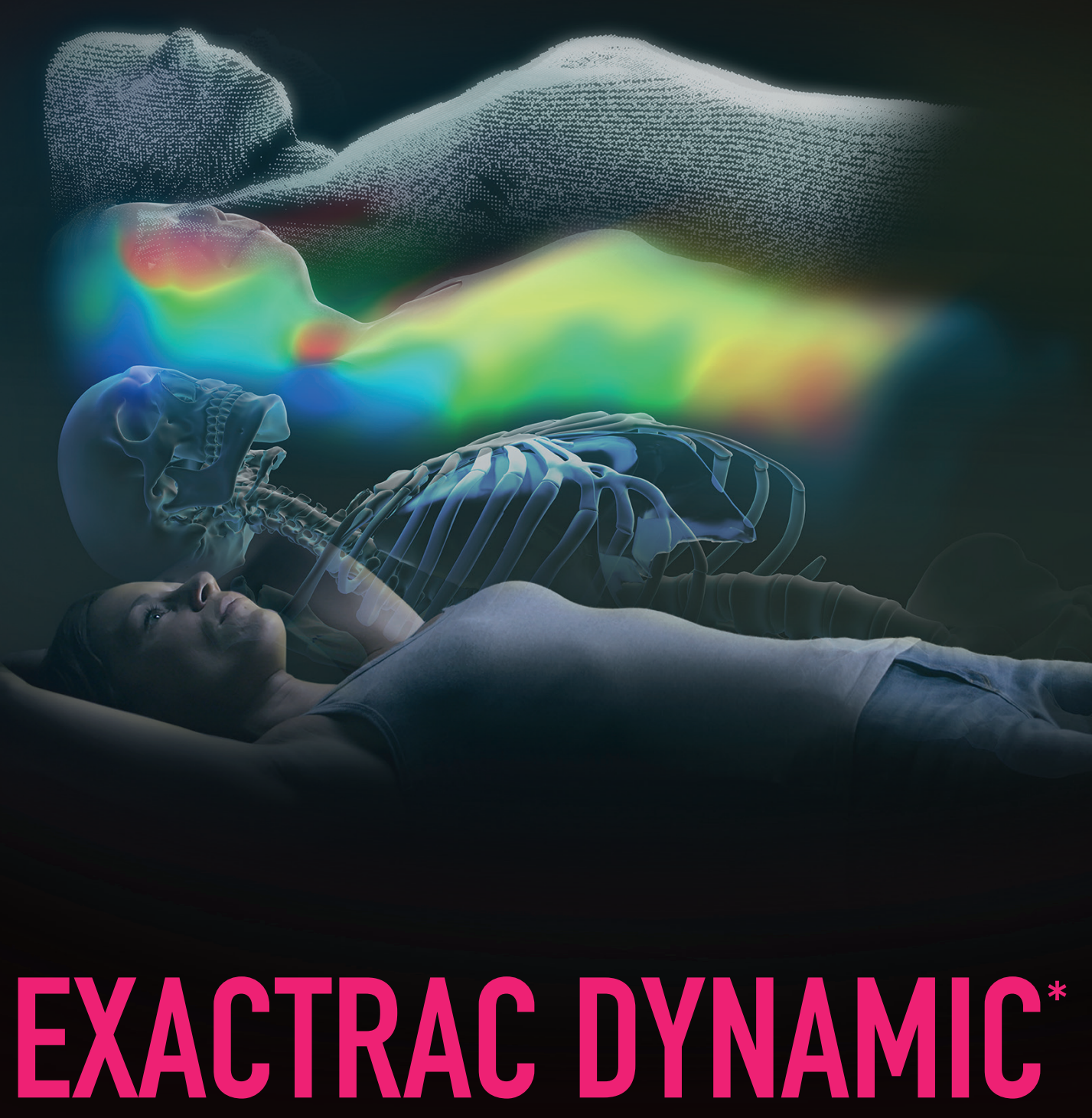

A New Dimension of Patient Positioning \& Monitoring

Learn how the next generation of ExacTrac is streamlining radiotherapy treatments for a broad range of clinical workflows at brainlab.com/exactrac. 